\title{
BMJ Open Across-subjects multiple baseline trial of exposure-based cognitive-behavioral therapy for severe irritability: a study protocol
}

\author{
Reut Naim (D) , Katharina Kircanski, ${ }^{1}$ Andrea Gold, ${ }^{2,3}$ Ramaris E German, ${ }^{1}$ \\ Mollie Davis, ${ }^{1}$ Samantha Perlstein, ${ }^{1}$ Michal Clayton, ${ }^{1}$ Olga Revzina, ${ }^{1}$ \\ Melissa A Brotman ${ }^{1}$
}

To cite: Naim R, Kircanski K, Gold A, et al. Acrosssubjects multiple baseline trial of exposure-based cognitive-behavioral therapy for severe irritability: a study protocol. BMJ Open 2021;11:e039169. doi:10.1136/ bmjopen-2020-039169

- Prepublication history for this paper is available online. To view these files, please visit the journal online (http://dx.doi org/10.1136/bmjopen-2020039169).

Received 10 April 2020 Revised 12 December 2020 Accepted 19 December 2020

Check for updates

(C) Author(s) (or their employer(s)) 2021. Re-use permitted under CC BY-NC. No commercial re-use. See rights and permissions. Published by BMJ.

${ }^{1}$ Emotion and Development Branch, National Institute of Mental Health, National Institutes of Health, Bethesda, Maryland, USA

${ }^{2}$ Pediatric Anxiety Research Center, Bradley Hospital, Riverside, Rhode Island, USA ${ }^{3}$ Department of Psychiatry \& Human Behavior, Warren Alpert Medical School of Brown University, Providence, Rhode Island, USA

Correspondence to

Dr Reut Naim; naimr2@nih.gov

\section{ABSTRACT}

Introduction Irritability is defined as a tendency towards anger in response to frustration. Clinically, impairing irritability is a significant public health problem. There is a need for mechanism-based psychotherapies targeting severe irritability as it manifests in the context of disruptive mood dysregulation disorder (DMDD). This study protocol describes a randomised multiple baseline design testing the preliminary efficacy of a new treatment, exposurebased cognitive-behavioral therapy for severe irritability in youth, which also integrates components of parent management training. We will investigate associations of this intervention with primary clinical measures, as well as ecological momentary assessment measures.

Methods and analysis Forty youth will be enrolled. Participants, aged 8-17 years, must present at least one of two core symptoms of DMDD: abnormal mood or increased reactivity to negative emotional stimuli, with severe impairment in one domain (home, school, peers) and moderate in another, or moderate impairment in at least two domains. Each participant is randomised to a 2-week, 4-week or 6-week baseline observation period, followed by 12 active treatment sessions. Clinical ratings are conducted at baseline, biweekly (clinician), weekly (parent/child) throughout treatment, post-treatment, and 3-month and 6-month follow-up (clinician). Clinician ratings on the Affective Reactivity Index and Clinical Global Impressions-Improvement scale for DMDD are our primary outcome measures. Secondary outcome measures include parent and child reports of irritability. Post hoc additional symptom measures include clinician, parent and self-ratings of depression, anxiety and overall functional impairment. Prospective, digitally based event sampling of symptoms is acquired for a week pretreatment, mid-treatment and post-treatment. Based on our pathophysiological model of irritability implicating frustrative non-reward, aberrant threat processing and instrumental learning, we probe these three brain-based targets using functional MRI paradigms to assess target engagement.

Ethics and dissemination The research project and all related materials were submitted and approved by the appropriate Institutional Review Board (IRB) of the National Institute of Mental Health (NIMH).
Strengths and limitations of this study

- This study protocol presents and tests a novel intervention to target core symptoms of disruptive mood dysregulation disorder (DMDD) symptoms (irritable mood, temper outbursts)

- This study protocol is employing parent management training (PMT) and principles of extinction derived from exposure therapy to target key mechanism of aberrant reward and threat processing, two core pathophysiologically informed dysfunctions posited in DMDD.

- The current protocol leverages state-of-the-art measures by collecting intensive, week-by-week clinical symptom data from patients, parents and trained clinicians, as well as behavioral and neural data from patients during the treatment.

- Despite the strengths and innovative aspects of the current study, the current multibaseline design is not a randomised controlled trial, therefore, it does not allow for strong causal inferences.

- The combination of both PMT and exposure techniques in the current treatment does not allow for separation of their effects.

Trial registration numbers NCT02531893 and NCT00025935.

\section{INTRODUCTION}

Irritability is defined as a tendency towards anger and outbursts. ${ }^{1}$ Clinically, impairing irritability is a significant public health problem, ${ }^{23}$ and is among the most frequent presenting problems in youth psychiatric practice. ${ }^{4-8}$ Longitudinally, irritability predicts depression, anxiety, ${ }^{9}{ }^{10}$ suicidality, ${ }^{2}{ }^{11}$ and is associated with functional impairment. ${ }^{5}$ The need to diagnose address and treat youth with severe impairing irritability led to the inclusion of disruptive mood dysregulation disorder (DMDD) in the Diagnostic and Statistical Manual of Mental Disorders-5th 
edition (DSM-5). While this decision was somewhat controversial, ${ }^{12}$ DMDD codifies clinically-significant temper outbursts and irritable mood. However, there is a need for additional evidence-based psychotherapies that specifically target the temper outbursts and irritable mood, the two core symptoms articulated in DMDD. ${ }^{13-15}$ Coupled with this need, in recent years, there has been a movement towards treatment innovation based on engaging brain-behaviour targets that are hypothesised as translational mechanisms. ${ }^{16}$ In this study, we test both clinical efficacy and target engagement of a cognitivebehavioral therapy (CBT) for severe irritability as it presents in DMDD. Only one prior treatment study targeting anger leverages pathophysiology to guide the treatment intervention. ${ }^{17}$ Thus, additional mechanism-based, targeted interventions for DMDD are needed. ${ }^{1618-25}$

Biomarkers have transformed modern medicine but remain largely elusive in psychiatry. To date, treatments have either been serendipitous and/or developed primarily to address specific clinical symptoms. Augmenting this mechanism-driven approach, researchers ${ }^{18} 19{ }^{26}$ have suggested that experimental therapeutics should engage pathophysiological targets, while synergistically leveraging prior symptom-based theoretical approaches. Thus, here, we integrate and test mechanism-based approaches to target two core deficits in impairing irritability ${ }^{1}$ : (1) aberrant reward processing and (2) aberrant threat processing. The inclusion of therapeutic techniques to target key mechanisms of irritability is intended to lead to more efficient, generalized and long-term outcomes. ${ }^{27}$

\section{Prior relevant treatments}

Some empirically supported interventions target irritability and disruptive behaviour as seen in disruptive behaviour disorders (DBD): attention-deficithyperactivity disorder (ADHD), oppositional defiant disorder (ODD), and conduct disorder. However, there are two opportunities to further build on this research. First, few existing interventions specifically target severe, impairing irritability and temper outbusts as present in the context of DMDD, which is a mood disorder as opposed to DBD. ${ }^{28} 29$ Second, while there are a few categories of existing relevant empirically supported interventions: parent management training (PMT) and CBT for DBD, and dialectical behaviour therapy for preadolescent children (DBT-C) and interpersonal therapy (IPT) for DMDD, such treatments do not emphasise exposure techniques as a primary target.

Meta-analytical evidence supports the efficacy of PMT in decreasing externalising behaviours, aggression, oppositionality and impulsivity characteristic of DBDs,${ }^{30}$ with a medium effect size for trials using blinded assessments. ${ }^{31}{ }^{32}$ For example, Parent-Child Interaction Therapy ${ }^{33}$ is a manualized parent training intervention that has received substantial empirical support in the treatment of disruptive behaviour. Other interventions, such as the Incredible Years, ${ }^{35}$ Stop now and $\operatorname{Plan}^{36}$ and Kazdin's PMT, ${ }^{37}$ are also evidence-based psychotherapies for externalising problems. However, most are targeted towards very young children ${ }^{30} 35$ or target a broad spectrum of externalising problems. ${ }^{36}$ Although some of these protocols include treating anger and aggressive outbursts ${ }^{35}{ }^{38-41}$ there is no protocol modified specifically to parents of youth with DMDD, potentially limiting the generalizability across symptom domains. Furthermore, studies of youth with ODD and ADHD have yet to systematically examine the effects of PMT on emotional symptoms, notably the irritable dimension. ${ }^{42}$ PMT efficacy studies are needed to investigate the unique clinical and pathophysiological features of severe irritability in children and adolescents. ${ }^{143}$ Early research suggests that youth with severe mood dysregulation show deficits during probabilistic response reversal and reversing previously rewarded associations. ${ }^{445}$ It remains unknown whether PMT addresses such deficits in reward learning.

CBT, which targets relationships among thoughts, behaviours and feelings ${ }^{46}$ has also shown efficacy in treating anger and disruptive behaviour. ${ }^{1729}$ Sukhodolsky et $a l^{17}$ developed a CBT protocol for aggression using three modules: emotion regulation, social problemsolving and the social skills training aimed to prevent and resolve conflict situations. The first module starts with identifying anger triggers, developing prevention strategies and learning emotion regulation skills, such as cognitive reappraisal and relaxation training. The second module focuses on problem-solving skills, such as generating multiple solutions and considering different consequences and actions when dealing with conflicts. The final module focuses on developing skills for preventing or resolving potentially anger-provoking situations in different domains.

In a meta-analysis of CBT for anger in youth, Sukhodolsky et $a l^{47}$ reported a medium mean effect size across parent, teacher, observer and self-reports for physical aggression and/or delinquency in boys. Other meta-analyses and review papers examined the effect of evidence-based CBT/psychosocial treatments for disruptive behaviour or externalising disorders, showing mixed results for efficacy ${ }^{4-51}$; however, the participants in those studies were not specifically referred for severe, impairing irritability. Although promising, such anger-based CBT protocols emphasise cognitive techniques (eg, generation of multiple solutions and the consideration of consequences for different courses of action in conflicts), rather than behavioral interventions. Several behavioral principles, such as exposure, might be explored in clinically irritable youth. Exposure techniques help patients to systematically approach aversive situations while resisting urges to avoid or escape. Such techniques teach patients to approach and tolerate triggering stimuli, despite their feared outcomes, using a graded hierarchy of exposure stimuli. ${ }^{5253}$ A recent promising clinical case report using the Unified Protocol for Transdiagnostic Treatment of Emotional Disorders in Children (UP-C) ${ }^{54}$ demonstates a positive outcome in the treatment of a child with anger 
and irritability ${ }^{55}$ UP-C, an emotion-focused transdiagnostic treatment for anger, consists of cognitive-behavioral techniques, encouraging patients to experience thoughts more mindfully and neutrally. In a recent pilot study, ${ }^{56}$ Hawks $e t$ al adapted the UP-C for the treatment of paediatric irritability in a sample of 19 children (ages 8-12) with primary presenting concerns of irritability and/or disruptive behaviours. Results supported the feasibility and acceptability of this treatment and provided preliminary evidence that such an approach may yield improved outcomes for symptoms of paediatric irritability and disruptive behaviours. Another recently published study by Evans $e t a \tilde{l}^{\tilde{7}}$ examined the effectiveness of a modular, transdiagnostic, behavioral/cognitive-behavioral intervention (MATCH), which is an existing flexible manual, for treating youth with severe irritability, compared with standard manualized treatments and usual care. Irritability was not specifically targeted in this larger study. MATCH showed the most consistent improvement in reducing irritability across multiple measurement schedules and informants. Taken together, the development and examination of expanded behavioral (eg, exposurebased) interventions targeting irritability requires further research, towards the goal of identifying active components effective in reducing irritability in youth with DMDD.

In a complementary line of work, Perepletchikova et $a p^{58}$ adapted DBT for preadolescent children (DBTC), drawing from $\mathrm{DBT}^{59}$ and its adolescent adaptation (DBT-A).$^{60}$ DBT-C provides youth and parents with skills training, coaching and therapy aimed to help families apply and generalize skills in mindfulness, distress tolerance, emotion regulation, interpersonal effectiveness, validation and behaviourism/reinforcement principles. An initial DBT-C randomised controlled trial (RCT) in a sample of youth with DMDD demonstrated efficacy. ${ }^{58}$ At post-treatment assessment, following 32 weeks of DBT training, children reported a significant increase in adaptive coping skills and significant decreases in depressive symptoms and problematic internalising behaviours. ${ }^{58}$ In additional work examining irritability in the context of mood disorders, a recent pilot $\mathrm{RCT}^{61}$ assessed the feasibilty and preliminary efficacy of an adapted version of IPT for mood and behaviour dysregulation in youth with DMDD. Results indicated decreased outbursts and irritability and improved interpersonal interacting after treatment. In sum, paediatric adaptations of DBT and IPT show positive preliminary results in youth with DMDD.

Collectively, accumulating evidence supports the efficacy of different treatment components of PMT, CBT, DBT and IPT in treating externalising behaviours, aggression, oppositionality, impulsivity and irritability across various diagnoses. This novel protocol intervention builds on prior treatments. First, the current protocol specifically targets youth's encoding of nonreward and threat stimuli in the context of frustration, and integrates approaches of CBT and PMT. Second, the current protocol emphasises in vivo behavioral exposure to anger-inducing stimuli, independent of skills training in the additional domains of distress tolerance, mindfulness, interpersonal effectiveness and validation strategies.

\section{The current study}

The current protocol relies on a pathophysiological framework with the aim to implement CBT and PMT techniques to adress pathophysiological targets. Specifically, the intervention is capitalising on empirically supported and theory-driven interventions and posits two core mechanisms of impairing irritability: (1) exposure-based CBT to target exaggerated responses to frustrative nonreward and/or threatening stimuli and (2) PMT to target aberrant reward processing; with both mechanisms being mediated by abnormalities in frontoamygdalar circuitry. Some studies show that high irritability is associated with decreased activation in regulatory prefrontal regions during frustration, ${ }^{62} 63$ and that these regions have a critical role in emotion processing by regulating activity of the amygdala. ${ }^{64}$ Of note, regulation of amygdala activity by the prefrontal cortex seems to be one of the key mechanisms underlying exposure techniques. ${ }^{52}$ Some evidence suggests that exposure might not be successful in treating proactive aggression ${ }^{47}$ (representing predatory attacks motivated by external reward). However, irritability is characterised by reactive aggression, ${ }^{15}$ meaning affectively driven aggressive responses to others' behaviour that is perceived as threatening or intentional.

Here, we extend prior work by investigating CBT for chronic, severe and impairing irritability and temper outbursts. This CBT uses both in-session and betweensession in vivo exposures to anger-inducing stimuli as the primary behavioral technique. Previous evidence suggests that effective behavioral interventions targeting the threat system may also be applied to the treatment of anger-based disorders. Therefore, this protocol examines whether exposure to stimuli that evoke frustration and anger effectively reduces irritability. Similar to exposure therapy for anxiety and fear disorders, we conceptualise that exposure therapy will help severely irritable youth to increase their anger tolerance (without engaging in aggressive behaviours), decreasing manifestations of irritability, temper outburts and irritable mood. A novel aspect of the exposure work in the current study involves patient and therapist creating an anger hierarchy associated with different anger-inducing triggers for the patient. During therapy sessions, the therapist explicitly tries to evoke anger, before challenging the child to tolerate circumstances and demands, or to shape the environment to not acquiesce/reinforce.

Our PMT intervention relies on the assumption that whether a child's difficulties in reward processing manifest through irritable behaviour is also dependent on the parental behaviours and parental contingency management. Hence, the current protocol consists of a modified PMT, including elements such as active ignoring and positive praise. ${ }^{65}$ The incorporation of these PMT strategies aims to counteract deficits in frustrative non-reward 
response and, in turn, improve the child's symptoms and distress. ${ }^{66}$

An open pilot trial of exposure-based CBT procedures for DMDD demonstrated initial feasibility. ${ }^{32}$ Preliminary results indicated a pre-treatment to post-treatment reduction in DMDD symptom severity, as measured by the Clinical Global Impressions-Severity and Improvement (CGI-S, CGI-I ${ }^{6768}$ scale and the Clinician-Rated Affective Reactivity Index (CL-ARI) ${ }^{69}$ This CL-ARI is a paediatric, semistructured interview assessing temper outbursts and irritable mood. In the open trial, independent raters were not blind to treatment status. The current protocol addresses this limitation, in which clinicians' expectations may bias results, by using a randomised, multiple baseline design that staggers treatment onset across individuals. ${ }^{547}$ Multiple baseline study designs are commonly used in cases where the dependent variable is not expected to return to baseline after the treatment had been applied. This design has a few strengths including increased confidence that changes in outcomes are a result of the intervention, if those outcomes occur across cohorts.

To this end, we designed a protocol focusing on 12 weeks of exposure-based CBT plus PMT. The first half of each session (30-45 min) involves the patient completing exposure exercises. Throughout the therapy, the work with the child focuses on exposure, generation of an anger hierarchy, and gradual in-session exposure, and includes motivational interviewing to address ambivalence regarding change. Sessions with parents (30-45 min) comprise psychoeducation on reward learning and parental contingency management; parents are coached to praise and acknowledge their child's adaptive behaviours, and to actively ignore maladaptive behaviours. Other PMT elements include giving commands, setting limits, increasing time spent on positive joint activities, and delivering intermittent rewards. In later sessions, the therapist works with the parents on how to tolerate their emotional distress in response to their child's irritability symptoms. In between sessions, child is practicing tolerating their own anger through exposure while the parents are asked to employ techniques and strategies they had learnt in sessions (See table 1A,B) and for more details).

We expect, that over the course of the treatment, using exposure and PMT techniques, patients will have an increased ability to tolerate their own anger and frustration, and an increased ability to regulate their feelings and behaviours in the context of frustrative non-reward or threatening triggers manifested through a decrease in irritable mood and temper outbursts. Overall, we hypothesise that the treatment will lead to decreased irritability (decreased irritable mood and temper outbursts) in response to frustration and to an overall decrease in illness severity. Given the importance of examining target engagement in treatment studies, ${ }^{16}{ }^{18}$ we probe the hypothesised neural targets throughout the treatment based on our pathophysiological model of irritability. ${ }^{1}$ Specifically, participants complete resting state and task-based functional magnetic resonance imaging
Table 1A Content of the exposure-based CBT child sessions

\begin{tabular}{|c|c|}
\hline Session & Session components \\
\hline \multirow{10}{*}{$\begin{array}{l}\text { Session } \\
1^{*}\end{array}$} & Structure of treatment \\
\hline & - Building rapport \\
\hline & - Psychoeducation and normalisation \\
\hline & $\begin{array}{l}\text { Treatment targets and definitions (child's common } \\
\text { words for anger, irritability and temper outbursts) }\end{array}$ \\
\hline & $\begin{array}{l}\text { What does the child's anger look like and feel } \\
\text { like? }\end{array}$ \\
\hline & $\begin{array}{l}\text { Motivational interviewing to build motivation for } \\
\text { exposures }\end{array}$ \\
\hline & $\begin{array}{l}\text { 'Readiness ruler' - gauging readiness and } \\
\text { increasing motivation }\end{array}$ \\
\hline & $\begin{array}{l}\text { Temperature ratings - understanding how to rate } \\
\text { events/triggers on the anger thermometer from } 0 \\
\text { (not at all angry) to } 10 \text { (very angry) }\end{array}$ \\
\hline & $\begin{array}{l}\text { Generating anger hierarchy-common anger- } \\
\text { inducing triggers from least to highest anger } \\
\text { inducing. }\end{array}$ \\
\hline & $\begin{array}{l}\text { Practice activity: activity schedule (log of event/ } \\
\text { situation, emotion and level) }\end{array}$ \\
\hline \multirow[t]{7}{*}{ Session 2} & Review previous session and activity schedule \\
\hline & $\begin{array}{l}\text { Psycho education and motivational interviewing } \\
\text { review }\end{array}$ \\
\hline & - 'Readiness ruler' check \\
\hline & $\begin{array}{l}\text { Function of anger, prevalence of irritability, stigma } \\
\text { reduction }\end{array}$ \\
\hline & $\begin{array}{l}\text { Ways to understand irritability: fight or flight, } \\
\text { brain/body, behaviour, family reactions to anger }\end{array}$ \\
\hline & - Continue to generate anger hierarchy \\
\hline & - Practice activity: activity schedule \\
\hline
\end{tabular}

Session 3 - Review activity schedule

- Operationalizing OK ways to show anger

- Rationale for exposures

- Continue to generate anger hierarchy

- Imaginal exposure

- Role play interventions

- Guidelines for exposures and examples

- Practice activity: antecedents, behaviour, consequences worksheet

Session Exposures (tailor exposures to the triggers in 4-10 the anger hierarchy and use exposure sheets to document anger ratings throughout exposure)

- Operationalizing OK ways to show anger

- As appropriate 'readiness ruler' to reassess motivation

$\begin{array}{ll}\text { Session } & \text { Exposures (tailor exposures to the triggers in } \\ \text { the } 11 & \text { documer hierarchy and use exposure sheets to } \\ & \text { Operationalizing OK ways to show anger } \\ & \text { As appropriate 'readiness ruler' to reassess } \\ & \text { motivation }\end{array}$

Continued 


\begin{tabular}{ll}
\hline Table 1A & Continued \\
\hline Session & Session components \\
\hline & Termination activities: discussion on termination \\
& and maintenance and after-care plan \\
Session & Termination activities: discussion on termination \\
$12^{*}$ & and review maintenance and after care plan \\
& Look back and discuss all the exposures \\
& conducted and lessons learnt \\
& Engage in a patient-preferred activity for final \\
& session (ie, collage representing salient features \\
& of the sessions and triggers that have been \\
& overcome) \\
& Ceremoniously provide child with certificate of \\
completion
\end{tabular}

*Parent(s) are present for these sessions. Parents may also be present for exposure throughout the other sessions as needed.

\begin{tabular}{ll} 
Table 1B & Order of topics for parent sessions \\
\hline Module & Topic \\
\hline 1 & Assessment and motivational interviewing \\
\hline 2 & Introduction to parent intervention \\
\hline 3 & Instrumental learning \\
\hline 4 & Praise/acknowledgement \\
\hline 5 & Active ignore \\
\hline 6 & Dealing with outbursts \\
\hline 7 & Commands \\
\hline 8 & Limit setting \\
9 & Pleasant interactions and unexpected rewards \\
\hline
\end{tabular}

*Parent(s) are present for these sessions. Parents may also be present for exposure throughout the other sessions as needed. CBT, cognitive-behavioural therapy.

(fMRI) scans at different time points. Three fMRI paradigms assessing frustration, attention to threat and reward learning, separately, are included. ${ }^{32} 4471$ These paradigms test two hypotheses. First, we hypothesise that pre-treatment patterns of neural task activation will differentiate responders and non-responders (i.e., predictors or moderators). In an exploratory manner, we examine if activation and behavioral dysfunction across tasks can be used to determine who is most likely to benefit from treatment. Second, we predict that clinical improvement from pre-treatment to post-treatment will be mediated by prefrontal, striatal and amygdala circuit changes (i.e., mediation).$^{32} 71$

\section{METHODS AND ANALYSIS}

The current study investigates exposure-based CBT and PMT for chronic, severe irritability and temper outbursts as they present in DMDD.

\section{Procedures}

Informed consent and assent are obtained from parents and youth, respectively, after complete description of study procedures and prior to study procedures. All participants are advised that research is voluntary and that they may withdraw participation at any time. Participants and parents complete a pre-treatment clinical evaluation onsite before being enrolled in the protocol. A trained and licensed masters-level or doctoral-level clinician conducts interviews with the participants and parents. We screen participants and follow them for several months prior to randomisation; any medication changes must be made prior to randomisation. We communicate to the family and the community treatment provider that once the child is randomised, the regimen cannot be changed unless there is an acute clinical need. Once patient is enrolled in the study, the child's prescriptions are written by a National Institute of Mental Health (NIMH) psychiatrist who is working with the study team.

\section{Recruitment}

Patients are recruited locally (Maryland, District of Columbia, Virginia; USA) through Institutional Review Board (IRB)-approved postcards, mailings, announcements, advertisements, postings, flyers and contacts. Recruitment strategies include postcards and newsletters via direct mail, electronic postings and announcements on approved websites, postings on bulletin boards or placed in centres, distribution of flyers at speaking engagements/meetings (outreach exhibits, advocacy group meetings, parent groups, professional meetings, association/trade meetings), local print publication advertisements, social media advertisements (Twitter, Facebook and Instagram) and on local parent, advocacy and providers/professional groups and study participant recruitment listservs. Contacts are made to selected physicians and advocacy support groups. At the beginning of the time of recruitment for the current study, another study examining the effect of interpretation bias training (IBT) in reducing DMDD symptoms was running in our lab. Potential participants recruited during this time were presented with both forms of therapy and could choose participating in one or both of these studies if they wish. Those who wish to do both started with IBT. Study recruitment commenced in September 2018. Recruitment is expected to be completed by 2022 .

\section{Inclusion/exclusion criteria}

Participants, ages 8-17 years, must be fluent in English and have an IQ above 70, as assessed by the Wechsler Abbreviated Scale of Intelligence (WASI). ${ }^{72}$ Participants must currently present at least one of two core symptoms of DMDD: abnormal mood or increased reactivity to negative emotional stimuli, with severe impairment in one domain (home, school, peers) and moderate in another, or moderate impairment in at least two domains. Symptoms and diagnoses are established using a semistructured clinical interview (Kiddie-Schedule for Affective Disorders and Schizophrenia Present and Lifetime Version; K-SADS-PL) ${ }^{73}$ with the additional DMDD supplement. ${ }^{74}$ Additionally, based on record review and 
interviews with the child and parent, the clinical research team must agree that if the child is in active treatment, the child's response to their current treatment is no more than minimal determined by a score of 3 or more on the CGI-S. Unless there is an acute clinical need, participants must have no planned changes in outpatient psychiatric treatment regimen, which can include any psychotropic medications and/or psychotherapeutic interventions, 2 weeks prior to enrollment and throughout the weeks of treatment and post-treatment assessment.

Participants are excluded if the irritability symptoms are due to the direct physiological effects of a drug, or to a general medical or neurological condition. Additionally, participants who meet DSM-5 criteria, as assessed by the K-SADS-PL, ${ }^{73}$ for schizophrenia, schizophreniform disorder, schizoaffective illness, autism spectrum disorder (ASD), major depressive episode, or post-traumatic stress disorder, are also excluded. ASD exclusion is operationalized using the Development and Well-Being Assessment (DAWBA) ${ }^{75}$ the Social Responsiveness Scale (SRS), ${ }^{76}$ the Social Communication Questionnaire $(\mathrm{SCQ})^{77}$ and the Children's Communication Checklist-Second Edition (CCC-2) ${ }^{78}$ Specifically, we use cut-off scores of $>75$ on SRS, $>15$ on SCQ and $<-15$ on CCC. When one of these scores is above cut-off, with a combination of a DAWBA score, that is, $\geq 4$, the participant is excluded. Participants are not eligible if they exhibit active suicidality or any cardinal bipolar symptoms, such as elevated or expansive mood, grandiosity or inflated self-esteem, and $\backslash$ or decreased need for sleep. Participants with other comorbid psychiatric problems (e.g., ADHD, anxiety disorders, past major depressive disorder; MDD) are not excluded from participation in this study.

\section{Study design}

Forty youth meeting inclusion/exclusion criteria will be enrolled. Table 2 provides a detailed schedule of enrollment and intervention as well as a list of each of the measures being collected at each time point in the trial timeline. Each participant undergoes a randomised baseline observation period of 2, 4 or 6 weeks, followed by active treatment, and finally, post-treatment and follow-up observation periods (at 3 months and at 6 months following treatment termination). The multiple baseline design of the study controls for overall effects of time and regression towards the mean. ${ }^{70}$ Rating clinicians (table 2) are blinded to randomisation status of the participant and the timepoint the participant is in the trial at the time of the rating to control for clinician expectations. Randomisation to the different baseline points is independent to whether or not participants completed IBT study prior to starting the current protocol. Follow-up clinician-reported, child-reported and parent-reported primary and secondary outcomes are obtained at 3 and 6 months post-treatment. All visits are at the NIMH. All participants receive the active treatment. ${ }^{79}$

Twelve weekly sessions last 60-90 min in an NIMH outpatient clinic, with both individual and joint child and parent meetings. There is one primary clinician treating the youth. Therapeutic procedures engaged in sessions are outlined in table 1A,B). ${ }^{32}{ }^{80}$ available upon request The manual involves a variety of exposure-based CBT procedures, such as creating hierarchies of irritabilityinducing events, exposure exercises (e.g., role-play, in vivo and imaginal), and PMT techniques (e.g., praise/ acknowledgement, pleasant interactions and unexpected rewards). ${ }^{298182}$ The exposures and the modules in the PMT are designed to be flexibly delivered and tailored for the specific issues and anger-inducing triggers identified by the individual child and their parents; therefore, the PMT modules will be adapted to account for age differences and will address the most salient problem areas identified using parent feedback.

The main clinical outcomes we plan to assess are changes in irritability and overall illness severity and improvement. Changes in irritability will be assessed biweekly by clinician raters. Illness severity will be assessed biweekly and improvement will be assessed at mid-treatment and posttreatment. Illness severity at the end of the treatment will be compared with pre-treatment severity to determine the treatment's impact on the patient's overall severity of illness and to ascertain a frequently used global designation of 'responder' status. ${ }^{83}$

The secondary clinical outcomes that will be assessed are child-reported and parent-reported irritability assessed weekly, and in vivo ecological momentary assessment (EMA) measures of mood and impairment, assessed pre-treatment, mid-treatment and post-treatment. Post hoc additional symptom measures include clinician-rated depressive and anxiety symptoms and functional impairment assessed biweekly. Additional secondary child-rated and parent-rated clinical outcomes include depressive and anxiety symptoms and aggression reported at pretreatment and post-treatment.

\section{Modifications made due to COVID-19}

Due to the circumstances of the ongoing COVID-19 pandemic, recruitment was paused for about 5 months (Mid-March to mid-August 2020). To accommodate the protocol, we are continuing to adhere to the manual and provide treatment remotely. For patients going through the trial when the stay-at-home orders started, we shifted to telehealth. Exposures continue to be conducted and, in some ways, have benefited from being able to use the home as context in the exposures and parents being more at home to use in the exposures. Scans had stopped since mid-March.

Procedures for administration of clinical, child and parent report measures, as well as EMA, stayed similar as to the rest of the trial, with the exception of the presession ARI allowed to be collected up to 2 days before the session for the pre-session, and postsession alliance measures allowed to be collected almost a week after the session. Self-report and parent report measures require more effort to assure compliance as these are collected remotely and via technology as opposed to on site as 
Table 2 Enrollment, assessment and intervention timeline

\section{Study period}

\section{Enrolment Randomisation treatment Post-randomisation}

\begin{tabular}{|c|c|c|c|c|c|c|c|c|c|}
\hline Timepoint & $-t_{1}$ & 0 & $\mathrm{t}_{1}$ & $\begin{array}{l}\text { Sessions } \\
1-6\end{array}$ & $\begin{array}{l}\text { Mid- } \\
\text { treatment }\end{array}$ & $\begin{array}{l}\text { Sessions } \\
7-12\end{array}$ & $\begin{array}{l}\text { Post- } \\
\text { treatment }\end{array}$ & $\begin{array}{l}3 \text { months } \\
\text { follow-up }\end{array}$ & $\begin{array}{l}6 \text { months } \\
\text { follow-up }\end{array}$ \\
\hline \multicolumn{10}{|l|}{ Enrollment } \\
\hline $\begin{array}{l}\text { Eligibility } \\
\text { screen }\end{array}$ & $x$ & & & & & & & & \\
\hline K-SADS-PL & $x$ & & & & & & & & \\
\hline WASI & $x$ & & & & & & & & \\
\hline DAWBA & $x$ & & & & & & & & \\
\hline $\mathrm{CCC}-2$ & $x$ & & & & & & & & \\
\hline \multicolumn{10}{|l|}{ SRS } \\
\hline SCQ & $x$ & & & & & & & & \\
\hline $\begin{array}{l}\text { Informed } \\
\text { consent }\end{array}$ & $x$ & & & & & & & & \\
\hline Allocation & & $x$ & & & & & & & \\
\hline \multicolumn{10}{|l|}{ Assessments } \\
\hline EMA & & & $x$ & & $x$ & & $x$ & & \\
\hline \multicolumn{10}{|c|}{ FMRI and scans tasks } \\
\hline Resting state & & & $x$ & & $x$ & & $x$ & & \\
\hline AP3 & & & $x$ & & $x$ & & $x$ & & \\
\hline Dot-Probe & & & $x$ & & & & $x$ & & \\
\hline Carnival & & & $x$ & & & & $x$ & & \\
\hline DTI & & & $x$ & & & & & & \\
\hline \multicolumn{10}{|c|}{ Clinician-rated measures } \\
\hline $\begin{array}{l}\text { CGI-S for } \\
\text { DMDD } \\
\text { (1 week } \\
\text { version) }\end{array}$ & & & $x$ & $\begin{array}{l}\text { x (bi- } \\
\text { weekly) }\end{array}$ & & x (bi-weekly) & $x$ & $x$ & $x$ \\
\hline $\begin{array}{l}\text { CGI-I for } \\
\text { DMDD }\end{array}$ & & & & & $x$ & & $x$ & $x$ & $x$ \\
\hline CL-ARI & & & $x$ & $\begin{array}{l}X \text { (bi- } \\
\text { weekly) }\end{array}$ & & x (bi-weekly) & $x$ & $x$ & $x$ \\
\hline CDRS & & & $x$ & $\begin{array}{l}\text { x (bi- } \\
\text { weekly) }\end{array}$ & & x (bi-weekly) & $x$ & $x$ & $x$ \\
\hline PARS & & & $X$ & $\begin{array}{l}\text { x (bi- } \\
\text { weekly) }\end{array}$ & & x (bi-weekly) & $x$ & $x$ & $x$ \\
\hline CGAS & & & $X$ & $\begin{array}{l}\text { x (bi- } \\
\text { weekly) }\end{array}$ & & x (bi-weekly) & $x$ & $x$ & $x$ \\
\hline $\begin{array}{l}\text { Adherence } \\
\text { rating }\end{array}$ & & & & x (weekly) & & x (weekly) & & & \\
\hline $\begin{array}{l}\text { Therapeutic } \\
\text { alliance rating }\end{array}$ & & & & x (weekly) & & x (weekly) & & & \\
\hline \multicolumn{10}{|c|}{ Parent-rated measures } \\
\hline Scared & & & $x$ & & & & $x$ & $x$ & $x$ \\
\hline $\begin{array}{l}\text { ARI } 1 \text { week } \\
\text { version }\end{array}$ & & & $x$ & x (weekly) & & x (weekly) & $x$ & $x$ & $x$ \\
\hline $\begin{array}{l}\text { ARI } 6 \text { months } \\
\text { version }\end{array}$ & & & $x$ & & $x$ & & $x$ & & \\
\hline MOAS & & & $x$ & & & & $x$ & $x$ & $x$ \\
\hline
\end{tabular}


Study period

Enrolment Randomisation $\begin{aligned} & \text { Pre } \\ & \text { treatment Post-randomisation }\end{aligned}$

\begin{tabular}{|c|c|c|c|c|c|c|c|}
\hline $\begin{array}{l}\text { Therapeutic } \\
\text { alliance rating }\end{array}$ & & \multicolumn{2}{|l|}{ x (weekly) } & \multicolumn{3}{|l|}{ x (weekly) } & \\
\hline \multicolumn{8}{|c|}{ Child-rated measures } \\
\hline MFQ & $x$ & & & & $x$ & $x$ & $x$ \\
\hline $\begin{array}{l}\text { ARI- } 1 \text { week } \\
\text { version }\end{array}$ & $x$ & x (weekly) & & x (weekly) & $x$ & $x$ & $x$ \\
\hline $\begin{array}{l}\text { Therapeutic } \\
\text { alliance rating }\end{array}$ & & x (weekly) & & x (weekly) & & & \\
\hline
\end{tabular}

AP3, Affective Posner; CCC-2, Children's Communication Checklist-Second Edition ; CDRS, Children's Depression Rating Scale; CGAS, Children's Global Assessment Scale; CGI-S/I, Clinical Global Impressions-Severity and Improvement; CL-ARI, Clinician-Rated Affective Reactivity Index; DAWBA, Development and Well-Being Assessment; DMDD, disruptive mood dysregulation disorder; DTI, diffusion tensor imaging; EMA, ecological momentary assessment; fMRI, functional MRI; K-SADS-PL, Kiddie-Schedule for Affective Disorders and Schizophrenia Present and Lifetime Version; MFQ, Mood and Feelings Questionnaire; MOAS, Modified Overt Aggression Scale; PARS, Paediatric Anxiety Rating Scale; SCARED, Screen for Childhood Anxiety Related Emotional Disorders; SCQ, Social Communication Questionnaire; SRS, Social Responsiveness Scale; WASI, Wechsler Abbreviated Scale of Intelligence.

before. For the clinician ARI, clinicians consider the new circumstances of virtual schooling and being more at home, when evaluating impairment at school, at home and with peers. To protect privacy and due to lack of feasibility, sessions are no longer being recorded.

While attempting to both, address some of the mental health burdens brought on by the pandemic and being compliant with protocol modules, it is challenging to address all the additional pandemic-related mental health difficulties such as kids' outbursts being more severe or parents changing their parenting style due to elevated stress. The potential effect of the pandemic on the results will be tested during data analysis, comparing and/or controlling the two groups of kids recruited before and after the pandemic outbreak.

\section{Discontinuation/modification criteria}

The criteria for discontinuing the trial include the patients' request to discontinue or if the participant's condition worsens. All participants are informed that they may discontinue participation at their request at any time and for any reason and that they will not lose any benefits to which they are otherwise entitled. Also, if a participant's condition worsens to where it would be deemed unsafe or clinically inappropriate for participation to continue as determined by the clinical team, then participant will be withdrawn from the trial. The patient's own care providers in the community would be contacted to discuss immediate care.

\section{Patient and public involvement}

For 2 years prior to the development of the treatment, informal focus groups were conducted with children with severe irritability to assess the priorities, potential experience and preferences of the children and family for whom the treatment was being developed. Questions during these informal meetings centred around preferred goals of the treatment, what parents and children were willing to do (questions on burden to participant and motivation), and identification of appropriate language for communications with the public to increase recruitment and interest. The research team also engaged in discussions with community providers during local and national presentations. The feedback from families and clinical professionals were geared towards making the treatment more generalizable, acceptable, feasible and equitable for the patient population under study.

\section{Measures}

Table 2 provides a clinical assessment timeline. In addition, treatment constructs are summarised in table 1B. Primary outcome measures include the $\mathrm{CL}_{-} \mathrm{ARI}^{69}$ to measure changes in irritability and CGI-Severity (CGIS) ${ }^{67} 68$ and CGI-Improvement (CGI-I) ${ }^{67} 68$ scales to measure overall illness severity and improvement, respectively. The CL-ARI is a 12 items semistructured interview for DMDD designed to be administered by a trained clinician (master's level or above) to parents and children. The CL-ARI total score is the weighted sum of three subscale scores: temper outbursts (range of 0-27), irritable mood between outbursts (range of $0-8$ ) and impairment (range of 0-15). Items are scored on Likert scales. Temper outburst frequency is scored on a 5-point scale, separately for mild, moderate and severe outbursts $(0=$ none, $4=$ more than one outburst every day). Temper outburst duration is scored on a 6 -point scale $(0=$ none, $5=60 \mathrm{~min})$. The irritable mood frequency item has a 
4 -point scale $(0=$ none, $3=$ four or more days). The irritable mood duration item queries whether irritable mood was present for most of the day (i.e., at least half the day with a yes/no response option), and is only completed if the irritable mood frequency rating is scored at the maximum score $(3=$ four or more days). The irritable mood severity item has a 6 -point scale $(0=$ not present, $5=$ severe). Last, impairment is rated separately in three different settings (family, school, peers) on 6-point scales $(0=$ none, $5=$ severe $)$. CL-ARI had a good reliabilty (Cronbach's $\alpha=0.89$ ) and validity scores. ${ }^{84}$

CGI-S is is a single-item, 7-point measure of overall DMDD severity ( $1=$ normal, $7=$ most extreme) over the past month. The CGI scales are commonly used in psychiatric clinical trials, for which they have established utility and reliability. ${ }^{85}$

Secondary outcome measures include parent and child report of irritability (child-rated and parent-rated ARI) ${ }^{86}$ Additionally, we probe other co-occurring phenotypes in this study, using clinician ratings of depressive symptoms (Children's Depression Rating Scale), ${ }^{87}$ anxiety symptoms (Paediatric Anxiety Rating Scale) ${ }^{88}$ and functional impairment (Children's Global Assessment Scale) ${ }^{89}$ Children and parents provide reports on the child's symptoms of anxiety (Screen for Childhood Anxiety Related Emotional Disorders-child, SCARED ${ }^{90}$; and SCAREDparent, ${ }^{90}$, respectively), aggression (Modified Overt Aggression Scale), ${ }^{91}$ and depression (Mood and Feelings Questionnaire, MFQ). ${ }^{92}$ All of these measures demonstrated good internal consistency and discriminant validity in previous studies.

Finally, EMA measures, a form of real-time digitally based event sampling are obtained to augment clinical phenotyping procedures, in addition to traditional clinical rating data. ${ }^{93}{ }^{94}$ Parents and children are prompted by smartphones for 7 days, three times per day, to answer questions about child symptoms and outbursts (occurring both, 'at the time of the beep' and 'since the last beep'), and functional impairment, and corresponded parental responses. The effect of the treatment on EMA measures will be assessed. Data quality of all measures in this study is continually monitored and checked postcollection.

\section{Treatment process measures}

Adherence measures were developed for this study to assess therapist fidelity and competence. With informed consent and assent, all treatment sessions are video recorded or audio recorded. Following each session, therapists rate themselves on adherence measures, and external raters blind to clinical outcome complete additional, observer ratings based on the recordings. Therapists, patients, parents and independent raters also assess the therapeutic alliance following every session. ${ }^{95} 96$ To improve adherence and minimise drift, group supervision facilitated by the developer of the treatment where the manual is reviewed is conducted weekly throughout the trial. Study therapists review each other's session recordings to provide feedback during supervision.

\section{Neuroimaging measures}

At pre-treatment, mid-treatment and post-treatment, participants complete resting state, ${ }^{97}$ as well as a frustration fMRI task (Affective Posner (AP3)). ${ }^{71}$ At pretreatment and post-treatment, participants complete a threat attention orienting task (Dot-Probe) ${ }^{32} 98$ and an instrumental reward learning task (Carnival) that also includes a frustration induction. Finally, diffusion tensor imaging, ${ }^{99} 100$ a structural neuroimaging measure of neural circuitry, is also collected pre-treatment to estimate the location, orientation and anisotropy of the brain's white matter tracts. Scans are completed within 3 weeks of pre-treatment (session 1) and post-treatment (session 12). To ensure that all participants have the same exposure to stimuli, participants who are unable or unwilling to scan (e.g., braces) complete these tasks behaviorally outside of the scanner.

\section{fMRI tasks}

\section{AP3 Task}

This task models frustrative non-reward by inducing, and then violating, the expectation of reward during an attention orienting task (see figure 1 for task overview). The task is organised into three blocks consisting of a series of timed-response trials in which participants identify a target following a cue (i.e., Posner task). ${ }^{71}$ At the end of each trial, children receive accuracy feedback, and, after the first block, win or lose money on each trial. On all trials, a black screen is presented between the target/ response and the feedback slide. During the first block, participants are told to respond as quickly as possible to the stimuli and that there is no reward or punishment, but they receive feedback on their accuracy. This block consists two non-frustration runs outside the scanner that set up the expectation for reward. During the second block, inside the scanner, participants are introduced to the monetary contingency and win or lose US $\$ 0.50$ when they make a correct or incorrect response, respectively. The third block, inside the scanner, is the frustration condition, during which feedback is rigged such that $60 \%$ of correct trials are followed by negative feedback and a loss of US\$0.50, and $40 \%$ of correct responses are followed by positive feedback and a gain of US\$0.50. Trials in which participants make incorrect responses are followed by accurate feedback. Finally, fixation trials, during which participants focus on a fixation mark in the centre of the screen, are included to facilitate comparisons between brain activity during task trials following wining/losing. At the end of each block, participants are asked to report the valence of their emotional response and their level of frustration. The AP3 task ${ }^{101-103}$ is a wellestablished task used in multiple studies, including in the context of DMDD, ${ }^{71} 104$ to investigate the circuitry mediating frustration. Previous studies demonstrate that in response to negative feedback received in the context of frustration, children with severe, chronic irritability are showing abnormal activations in regions implicated in emotion, attention, and reward processing. 


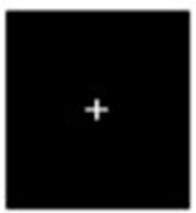

jitter ITI (500-5000ms; $\mathrm{M}=3000 \mathrm{~ms}$ )

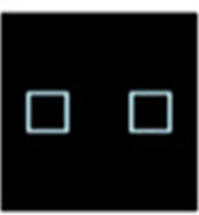

$300 \mathrm{~ms}$

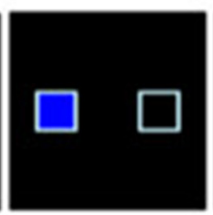

$200 \mathrm{~ms}$

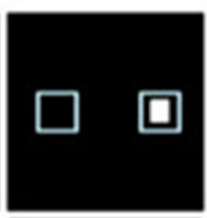

RESPONSE

$1260 \mathrm{~ms}$

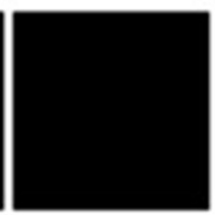

jitter ISI (1000$3000 \mathrm{~ms}$; $\mathrm{M}=2000 \mathrm{~ms}$ )

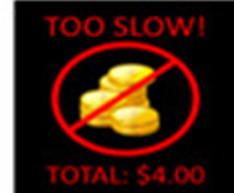

$60 \%$ correct

responses

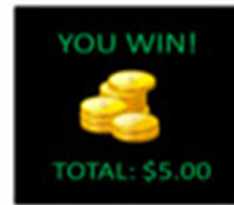

$40 \%$ correct responses

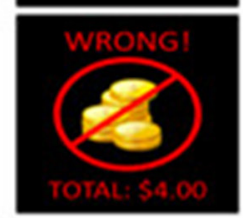

$2000 \mathrm{~ms}$
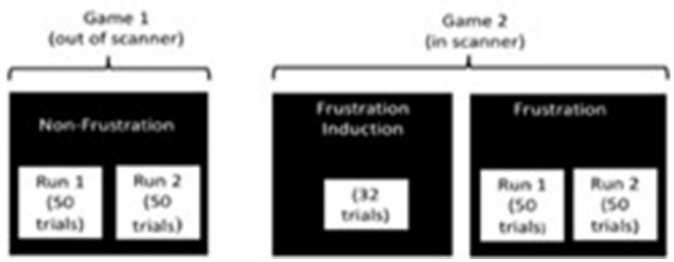

Figure 1 Affective posner (AP3) task overview.

\section{Dot-Probe Task}

This task measures threat-related attention orientation. There is an expanding literature using this task showing threat-related $^{105}$ attention bias in samples with high levels of anxiety and irritability. To date, fMRI research using the dot-probe task yields generally consistent findings of individual differences in anxiety related to perturbed function in brain regions supporting emotional processing (e.g., amygdala) and attentional control (e.g., ventrolateral prefrontal cortex, vlPFC; dorsolateral prefrontal cortex, dlPFC). ${ }^{98}$ In the current study, participants complete a faces-based variant of the dot-probe task, ${ }^{106}$ (following the TAU-NIMH ABMT Initiative protocol (http://people. socsci.tau.ac.il/mu/anxietytrauma/research/). The task includes 120 trials: 80 threat-neutral and 40 neutralneutral (see figure 2 for task trial (see figure 2 for task overview). In each trial, a pair of faces appears following a fixation cross. Following the face display, a probe appears on the screen (i.e., '<' or '>') in the location of a previously viewed either neutral (i.e., incongruent trials) or threatening face (i.e., congruent trials). Participants are instructed to indicate the orientation of the probe by clicking the left or right button of a button box. The probe remains on screen until a response is registered, and then the next trial begins. Attention bias to threat scores are calculated from reaction times (RTs) on the threat-neutral face display trials by subtracting congruent trial RTs from incongruent trial RTs. ${ }^{98}$ Positive attention bias to threat scores reflect a bias towards threat; negative scores reflect a bias away from threat. The Dot-Probe task starts with a resting state phase measuring pre-task brain activity at rest. ${ }^{107}$

\section{Carnival task}

This task is designed to examine reward vs loss learning in relation to irritability, both at baseline and following an acute frustration induction, and to investigate neural substrates involved in these processes. There are no previous studies examining reward learning per se in the context of DMDD. Related studies showed striatal dysfunction in the context of reward learning as compared with healthy youth. ${ }^{404}$ Youth with conduct problems, showed
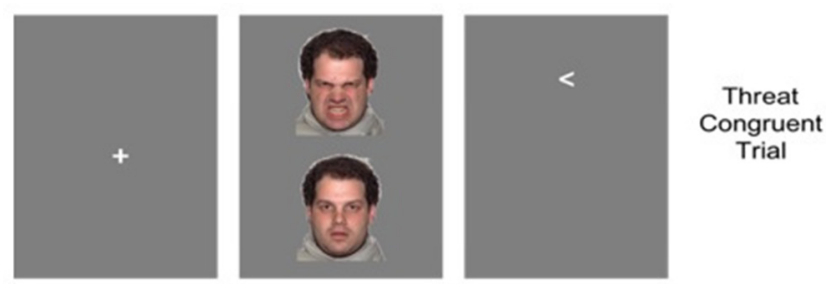

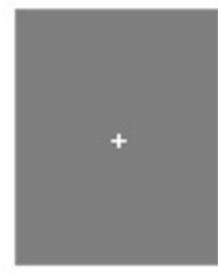

$500 \mathrm{~ms}$

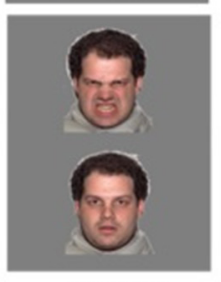

$500 \mathrm{~ms}$

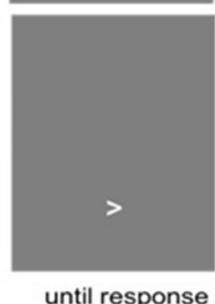

until response taken from the Nimstim Set (Tottenham ${ }^{115}$ et al (2009) that is available to the public. We followed the terms and conditions listed for using the set and agreed to use the images for research purposes only. The manuscript includes models that are listed as ones which are permitted to be published in scientific journals (see this link for more details: https://www. macbrain.org/resources.htm). 


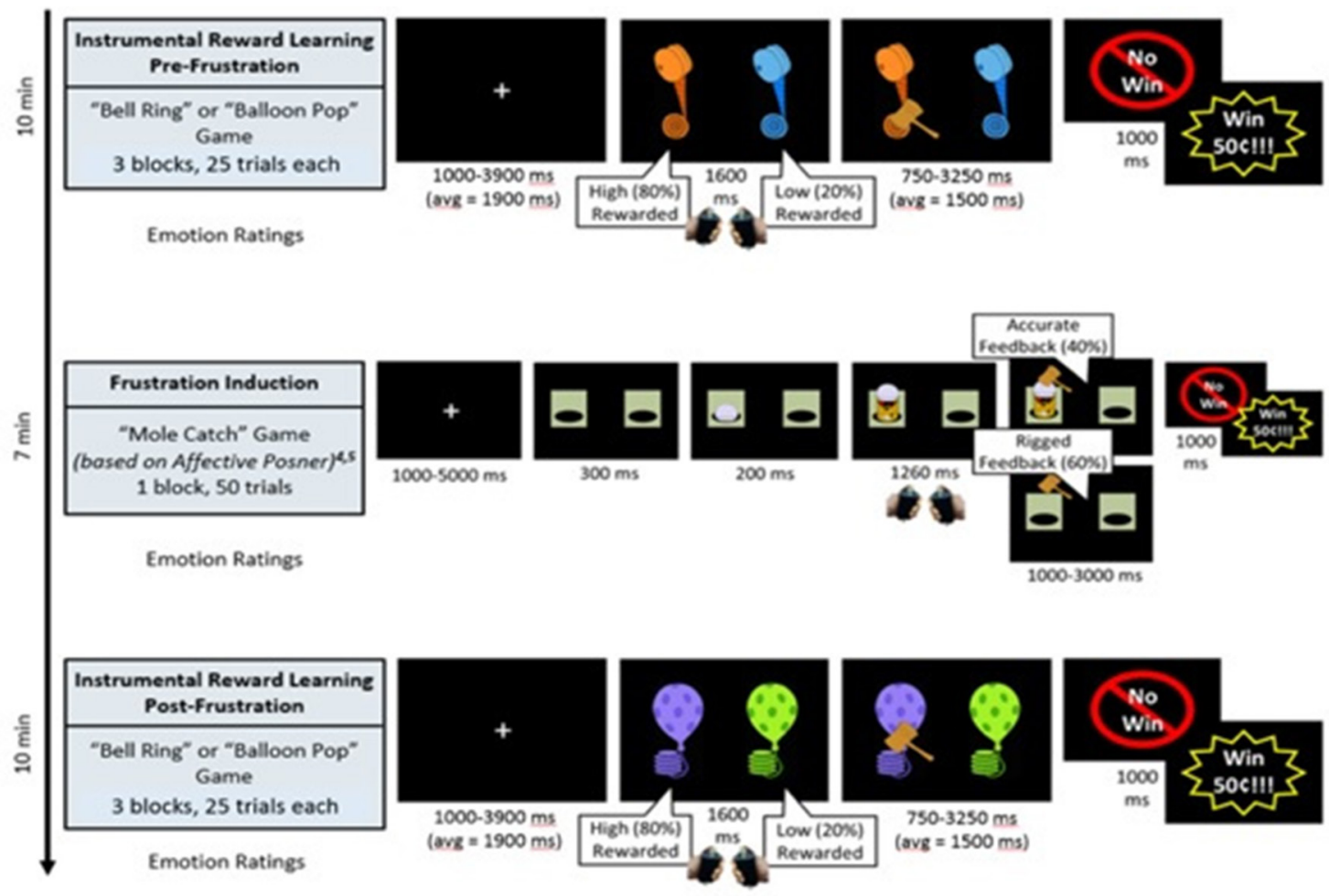

Figure 3 Carnival instrumental learning task overview.

atypical modulation of striatal activity based on stimulus expected value. ${ }^{108}$ The task simulates a set of Carnival games and has three phases (see figure 3 for task overview). In the first and last phases, participants view pairs of objects on the left and right sides of the computer screen, respectively. The paired stimuli are in different colours, and switch their location on the screen randomly. Participants hold a grip force response device (dynamometer) in each hand, and grip one of the devices while the stimuli are on the screen in order to select that stimulus in an attempt to obtain a monetary reward (US\$0.50). After participants respond, the screen acknowledges their selection by showing an image of a mallet overlaid on the selected stimulus. Next, a feedback screen appears indicating whether the selected stimulus is rewarded: 'Win $50 \not$ !' or 'No Win.' During this phase, participants learn over the course of three different sets of paired stimuli to select the stimulus that is followed more frequently by reward. In the second or middle phase, participants view images on the screen that simulate a whack-a-mole game. For $40 \%$ of participants' correct responses, the selection is followed by a reward ('Win 504!'). For the other $60 \%$ of correct responses, feedback is rigged, or followed by no reward ('No Win'). Incorrect responses are always followed by no reward. All images are original to the task. At the end of each phase, participants rate their levels of frustration, excitement and unhappiness. ${ }^{71}$

\section{Baseline assignment}

Baseline assignment sequence is created via a computerbased random number generator. Randomisation is performed in blocks of 10 participants with a 1:1 ratio within blocks. Rating clinicians are blind as to the current trial phase at time of ratings, as well as the baseline condition assignment. The blind is not broken for the entire cohort until completion of the trial.

\section{Sample size and power analysis}

The sample size of this study is expected to include $\mathrm{N}=40$ completers. To account for dropout, we have determined the total sample size of enrolment to be $\mathrm{N}=60$. Medium to large effects $(d=0.6-1.2)$ were detected in the pilot treatment conducted in our group using the current manual. ${ }^{32}$ There is no standard power analysis for multiple baseline designs. However, prior studies using this design with similar effect sizes generally have enrolled 6-18 participants. ${ }^{109} 110$ Various meta-analyses estimate the effect size of the psychological treatment/psychological placebo difference to be $0.40-0.60 .^{83}$ A sensitivity-power analyses using $\mathrm{G}^{*}$ Power v3.1 showed that with 40 participants, an error rate of 0.05 and a power of 0.95 , a large effect size of $d=0.81$ is required, using within-subjects analyses, while accounting multiple comparisons corrections. ${ }^{111}$ Since in the current study all participants receive an active treatment, we expect the effect size to be above and beyond placebo effect reported in the literature, and to be large in magnitude.

We will use intention-to-treat analyses for noncompliance and missing outcomes, using last observation carried forward approach. ${ }^{112}$

\section{Data analysis}

We will test the null hypothesis of no difference in DMDD symptoms improvement between time points across the randomised baseline schedules. Both visual inspection 
of the data and multilevel modelling in which repeated trials are nested within each subject will be used. ${ }^{113}$ Mixed models will be performed to examine symptom change over time for each of the ARI measures (clinician, child and parent rated) and the clinician-rated anxiety and depressive symptom measures. The slopes of the baseline phase will be modelled and compared with the slopes of the treatment phase to identify differential slopes between phases. Effect sizes will be calculated to assess if the treatment is associated with symptom improvement in clinician-rated severity of illness and functioning, and parent and child rated anxiety, depression and aggression. In addition to calculating statistical significance for all inferential tests, we will calculate clinically significant change for ARI measures and secondary outcome measures, using the method recommended by Jacobson and Truax. ${ }^{114}$ CGI-I, a measure of improvement, will also be used to determine clinical change.

For EMA ratings, we will use multilevel modelling to evaluate a pre-to-post-treatment increase in parental rewards for adaptive child behaviour, and decrease in rewards for irritable behaviour. Although the current design does not allow us to measure adjunctive versus separate effects of PMT vs exposure, based on sessions recording, we will calculate the dosage of each intervention at the individual level, and measure associations between intervention type proportion and treatment efficacy/clinical change. fMRI analyses will test for pre-treatment vs post-treatment differences in frontostriatal engagement after frustrative nonreward (AP3 and Carnival) and in frontostriatal-amygdala engagement during threat processing (Dot-probe). For each task, fMRI analysis will also compare pre-treatment and post-treatment resting state connectivity. For the AP3 task, we will compare activation on the $\mathrm{N}+1$ trial after rigged versus positive feedback. The AP3 task will also be used to examine whether neural changes at mid-treatment predate or mediate clinical symptom change at post-treatment. Resting state data will explore changes in default mode, attentional and salience networks over the course of treatment. For each task, we will calculate separateanalyses of variance for CGI-I and CL-ARI to test post-treatment ratings $\times$ task condition $\times$ time (scan 1,2) interactions. The model for CL-ARI will covary pre-treatment ratings.

\section{ETHICS}

To participate, parents must give written informed consent and the children must provide written assent. The research project and all related materials were submitted and approved by the appropriate IRB of the NIMH, which deemed the study to involve minimal risks. Adverse experiences include mild psychological distress and discomfort when responding to interview questions or questionnaires, engaging in the exposure-based treatment, or boredom during scanning or tasking. The principal and associate investigators monitor patients closely (e.g., at least weekly) throughout their participation in the study.

\section{Data management and monitoring}

Questionnaires and clinical ratings' data are managed through an IRB-approved password-protected institutional secure database, NIH Clinical Trials Database. Only approved NIMH staff have access to these data. Hard copy data/records with identifiers are double locked. Data for EMA is collected using secure technology, with procedures approved by the NIH's Information Systems Security Officer.

Data and safety are monitored by the principal investigator with a focus on clinical outcome, medication management and adverse events (AE). Data are also monitored by an independent data safety monitor from the NIH. The NIH's Science Review committee deemed that a data safety monitoring committee was not needed given the minimal risk category of the study and the capacity for the clinical staff and research team to closely monitor participants. Annual continuing reviews are conducted by the NIH's IRB to determine continuation of the research. Any serious AE will be reviewed and handled by the principal investigator, independent monitor and IRB. Any protocol modification must first be submitted as an amendment to the IRB and approved before their implementation. The research protocol is audited by the institution's Office of Regulatory Oversight to ensure compliance with Good Clinical Practice, organisational policies and regulations.

\section{Dissemination}

The study results will be disseminated through publications in peer-reviewed journals, presentations at national and international conferences, presentations at local school districts PTAs, presentations to community providers and to local advocacy and professional group organisations, and posted on the lab's website and through a lab newsletter that will be distributed to all lab and study participants.

Contributors $M A B$ is the principal investigator for this project. All authors contributed. MAB, KK, AG conceptualise the initial study design. RN wrote the first and successive drafts of the manuscript. KK, AG, REG, MD, SP, MC and OR contributed to writing and revising the manuscript. All authors read and approved the final manuscript.

Funding The study described in this study protocol is financially supported by the NIMH intramural program (ZIAMH002786-15, ZIAMH002778-17), conducted under NIH Clinical Study Protocols 15-M-0182 (ClinicalTrials.gov identifier: NCT02531893).

Disclaimer The funding body had no role in the study design, writing the manuscript, or the decision to submit the paper for publication. The study has not received specific, external funding.

Competing interests None declared.

Patient and public involvement Patients and/or the public were involved in the design, or conduct, or reporting, or dissemination plans of this research. Refer to the Methods section for further details.

Patient consent for publication Not required.

Provenance and peer review Not commissioned; externally peer reviewed.

Open access This is an open access article distributed in accordance with the Creative Commons Attribution Non Commercial (CC BY-NC 4.0) license, which permits others to distribute, remix, adapt, build upon this work noncommercially, and license their derivative works on different terms, provided the original work is properly cited, appropriate credit is given, any changes made 
indicated, and the use is non-commercial. See: http://creativecommons.org/ licenses/by-nc/4.0/.

ORCID iD

Reut Naim http://orcid.org/0000-0002-0424-5094

\section{REFERENCES}

1 Brotman MA, Kircanski K, Stringaris A, et al. Irritability in youths: a translational model. Am J Psychiatry 2017;174:520-32.

2 Orri M, Galera C, Turecki G, et al. Pathways of association between childhood irritability and adolescent suicidality. J Am Acad Child Adolesc Psychiatry 2019;58:99-107.

3 Stringaris A, Vidal-Ribas P. Probing the irritability-suicidality nexus. J Am Acad Child Adolesc Psychiatry 2019;58:18-19.

4 Dougherty LR, Smith VC, Bufferd SJ, et al. DSM-5 disruptive mood dysregulation disorder: correlates and predictors in young children. Psychol Med 2014;44:2339-50.

5 Copeland WE, Angold A, Costello EJ, et al. Prevalence, comorbidity, and correlates of DSM-5 proposed disruptive mood dysregulation disorder. AJP 2013;170:173-9.

6 Axelson D, Findling RL, Fristad MA, et al. Examining the proposed disruptive mood dysregulation disorder diagnosis in children in the longitudinal assessment of manic symptoms study. J Clin Psychiatry 2012;73:1342-50.

7 Margulies DM, Weintraub S, Basile J, et al. Will disruptive mood dysregulation disorder reduce false diagnosis of bipolar disorder in children? Bipolar Disord 2012;14:488-96.

8 Benarous X, Consoli A, Guilé J-M, et al. Evidence-Based treatments for youths with severely dysregulated mood: a qualitative systematic review of trials for SMD and DMDD. Eur Child Adolesc Psychiatry 2017;26:5-23.

9 Brotman MA, Schmajuk M, Rich BA, et al. Prevalence, clinical correlates, and longitudinal course of severe mood dysregulation in children. Biol Psychiatry 2006;60:991-7.

10 Vidal-Ribas P, Brotman MA, Valdivieso I, et al. The status of irritability in psychiatry: a conceptual and quantitative review. J Am Acad Child Adolesc Psychiatry 2016;55:556-70.

11 Pickles A, Aglan A, Collishaw S, et al. Predictors of suicidality across the life span: the Isle of Wight study. Psychol Med 2010;40:1453-66.

12 Avasthi A, Sarkar S, Grover S. Approaches to psychiatric nosology: a viewpoint. Indian J Psychiatry 2014;56:301-4.

13 Brotman MA, Kircanski K, Leibenluft E. Irritability in children and adolescents. Annu Rev Clin Psychol 2017;13:317-41.

14 Leibenluft E. DSM-10: coming soon to a psychiatric practice near you. J Am Acad Child Adolesc Psychiatry 2011;50:972-4.

15 Leibenluft E, Stoddard J. The developmental psychopathology of irritability. Dev Psychopathol 2013;25:1473-87.

16 Insel TR, Gogtay N. National Institute of mental health clinical trials: new opportunities, new expectations. JAMA Psychiatry 2014;71:745-6.

17 Sukhodolsky DG, Smith SD, McCauley SA, et al. Behavioral interventions for anger, irritability, and aggression in children and adolescents. J Child Adolesc Psychopharmacol 2016;26:58-64.

18 Insel TR. Next-Generation treatments for mental disorders. Sci Trans/ Med 2012;4:155ps19.

19 Gordon J. An experimental therapeutic approach to psychosocial interventions. NIMH Director's Message 2017.

20 Bzdok D, Varoquaux G, Steyerberg EW. Prediction, not association, paves the road to precision medicine. JAMA Psychiatry 2021;78:127.

21 White LK, Sequeira S, Britton JC, et al. Complementary features of attention bias modification therapy and cognitive-behavioral therapy in pediatric anxiety disorders. Am J Psychiatry 2017;174:775-84.

22 Friston KJ, Redish AD, Gordon JA. Computational nosology and precision psychiatry. Comput Psychiatr 2017;1:2-23.

23 Gordon JA. Medicine and the mind. N Engl J Med 2020;382:878-9.

24 Hyman SE. Revolution stalled. Sci Transl Med 2012;4:155cm11.

25 Insel TR. The NIMH experimental medicine initiative. World Psychiatry 2015;14:151-3.

26 Drysdale AT, Grosenick L, Downar J, et al. Resting-State connectivity biomarkers define neurophysiological subtypes of depression. Nat Med 2017;23:28-38.

27 Cook SC, Schwartz AC, Kaslow NJ. Evidence-Based psychotherapy: advantages and challenges. Neurotherapeutics 2017;14:537-45.

28 Barkley RA. Defiant children: A clinician's manual for assessment and parent training. 3rd edn. New York: Guilford Press, 2013.
29 Kazdin AE. Problem-solving skills training and parent management training for oppositiona defiant disorder and conduct disorder.. In: Evidence-Based psychotherapies for children and adolescents. 2nd edn. New York: The Guilford Press, 2010: 211-26.

30 Comer JS, Chow C, Chan PT, et al. Psychosocial treatment efficacy for disruptive behavior problems in very young children: a meta-analytic examination. J Am Acad Child Adolesc Psychiatry 2013;52:26-36.

31 Stringaris A, Vidal-Ribas P, Brotman MA, et al. Practitioner review: definition, recognition, and treatment challenges of irritability in young people. J Child Psychol Psychiatr 2018;59:721-39.

32 Kircanski K, Clayton ME, Leibenluft E, et al. Psychosocial treatment of irritability in youth. Curr Treat Options Psych 2018;5:129-40.

33 Eyberg SM, Robinson EA. Parent-child interaction training - effects on family functioning. J Clin Child Psychol 1982;11:130-7.

34 Zisser AR, Eyberg SM. Maternal ADHD: parent-child interactions and relations with child disruptive behavior. Child Fam Behav Ther 2012;34:33-52.

35 Menting ATA, Orobio de Castro B, Matthys W. Effectiveness of the Incredible years parent training to modify disruptive and prosocial child behavior: a meta-analytic review. Clin Psychol Rev 2013;33:901-13.

36 Augimeri LK, Farrington DP, Koegl CJ, et al. The SNAPTM under 12 outreach project: effects of a community based program for children with conduct problems. J Child Fam Stud 2007;16:799-807.

37 Kazdin AE. Parent management training and problem-solving skills training for child and adolescent conduct problems. In: Weisz JR, Kazdin AE, eds. Evidence-based psychotherapies for children and adolescents. 3rd edn. New York: Guilford Press, 2017.

38 Wyatt Kaminski J, Valle LA, Filene JH, et al. A meta-analytic review of components associated with parent training program effectiveness. J Abnorm Child Psychol 2008;36:567-89.

39 McCart MR, Priester PE, Davies WH, et al. Differential effectiveness of behavioral parent-training and cognitive-behavioral therapy for antisocial youth: a meta-analysis. J Abnorm Child Psychol 2006;34:525-41.

40 Michelson D, Davenport C, Dretzke J, et al. Do evidence-based interventions work when tested in the "Real world?" A systematic review and meta-analysis of parent management training for the treatment of child disruptive behavior. Clin Child Fam Psychol Rev 2013;16:18-34

41 Thomas R, Zimmer-Gembeck MJ. Behavioral outcomes of parentchild interaction therapy and triple $\mathrm{P}-$ Positive parenting program: a review and meta-analysis. J Abnorm Child Psychol 2007;35:475-95.

42 Stringaris A, Goodman R. Longitudinal outcome of youth oppositionality: irritable, headstrong, and hurtful behaviors have distinctive predictions. J Am Acad Child Adolesc Psychiatry 2009:48:404-12.

43 Blair RJ. The neurobiology of impulsive aggression. J Child Adolesc Psychopharmacol 2016;26:4-9.

44 Adleman NE, Kayser R, Dickstein D, et al. Neural correlates of reversal learning in severe mood dysregulation and pediatric bipolar disorder. J Am Acad Child Adolesc Psychiatry 2011;50:1173-85.

45 Dickstein DP, Nelson EE, McCLURE EB, et al. Cognitive flexibility in phenotypes of pediatric bipolar disorder. J Am Acad Child Adolesc Psychiatry 2007:46:341-55.

46 Beck JS. Cognitive behavior therapy: basics and beyond. 2nd edn. New York: Guilford Press, 2011.

47 Sukhodolsky DG, Kassinove H, Gorman BS. Cognitive-Behavioral therapy for anger in children and adolescents: a meta-analysis. Aggress Violent Behav 2004;9:247-69.

48 Battagliese G, Caccetta M, Luppino OI, et al. Cognitive-Behavioral therapy for externalizing disorders: a meta-analysis of treatment effectiveness. Behav Res Ther 2015;75:60-71.

49 Eyberg SM, Nelson MM, Boggs SR. Evidence-Based psychosocial treatments for children and adolescents with disruptive behavior. $J$ Clin Child Adolesc Psychol 2008;37:215-37.

50 Kaminski JW, Claussen AH. Evidence base update for psychosocia treatments for disruptive behaviors in children. J Clin Child Adolesc Psychol 2017;46:477-99.

51 McCart MR, Sheidow AJ. Evidence-Based psychosocial treatments for adolescents with disruptive behavior. J Clin Child Adolesc Psychol 2016;45:529-63.

52 Craske MG, Kircanski K, Zelikowsky M, et al. Optimizing inhibitory learning during exposure therapy. Behav Res Ther 2008;46:5-27.

53 Foa EB, McLean CP. The efficacy of exposure therapy for anxietyrelated disorders and its underlying mechanisms: the case of OCD and PTSD. Annu Rev Clin Psychol 2016;12:1-28.

54 Barlow DH, Nock M, Hersen M. Single case experimental designs: strategies for studying behavior for change, 2009. 
55 Grossman RA, Ehrenreich-May J. Using the unified protocol for Transdiagnostic treatment of emotional disorders with youth exhibiting anger and irritability. Cogn Behav Pract 2020;27:184-201.

56 Hawks JL, Kennedy SM, Holzman JBW, et al. Development and application of an innovative Transdiagnostic treatment approach for pediatric irritability. Behav Ther 2020;51:334-49.

57 Evans SC, Weisz JR, Carvalho AC, et al. Effects of standard and modular psychotherapies in the treatment of youth with severe irritability. J Consult Clin Psychol 2020;88:255-68.

58 Perepletchikova F, Nathanson D, Axelrod SR, et al. Randomized clinical trial of Dialectical behavior therapy for preadolescent children with disruptive mood dysregulation disorder: feasibility and outcomes. J Am Acad Child Adolesc Psychiatry 2017;56:832-40.

59 Linehan MM. Skills training manual for treating borderline personality disorder. New York: Guilford Press, 1993.

60 Miller AL, Rathus JH, Linehan MM. Dialectical behavior therapy with suicidal adolescents. New York: Guilford Press, 2007.

61 Miller L, Hlastala SA, Mufson L, et al. Interpersonal psychotherapy for mood and behavior dysregulation: pilot randomized trial. Depress Anxiety 2018;35:574-82.

62 Grabell AS, Li Y, Barker JW, et al. Evidence of Non-Linear Associations between Frustration-Related Prefrontal Cortex Activation and the Normal:Abnormal Spectrum of Irritability in Young Children. J Abnorm Child Psychol 2018;46:137-47.

63 Perlman SB, Jones BM, Wakschlag LS, et al. Neural substrates of child irritability in typically developing and psychiatric populations. Dev Cogn Neurosci 2015;14:71-80.

64 Motzkin JC, Philippi CL, Wolf RC, et al. Ventromedial prefrontal cortex is critical for the regulation of amygdala activity in humans. Biol Psychiatry 2015;77:276-84.

65 Hayes AM, Harris MS. The development of an integrative therapy for depression. Stress, Coping, and Depression 2000:291-306.

66 Forehand R, Jones DJ, Parent J. Behavioral parenting interventions for child disruptive behaviors and anxiety: what's different and what's the same. Clin Psychol Rev 2013;33:133-45.

67 Towbin K, Vidal-Ribas P, Brotman MA, et al. A double-blind randomized placebo-controlled trial of citalopram adjunctive to stimulant medication in youth with chronic severe irritability. $J A m$ Acad Child Adolesc Psychiatry 2020;59:350-61.

68 Walkup JT, Labellarte MJ, Riddle MA, et al. Fluvoxamine for the treatment of anxiety disorders in children and adolescents. $N$ Engl $J$ Med Overseas Ed 2001;344:1279-85.

69 Haller SP, Kircanski K, Stringaris A. Psychometric properties of the clinician affective reactivity index. Behavior Therapy.

70 Ferron J, Sentovich C. Statistical power of randomization tests used with multiple-baseline designs. J Exp Edu 2002;70:165-78.

71 Tseng W-L, Deveney CM, Stoddard J, et al. Brain mechanisms of attention orienting following frustration: associations with irritability and age in youths. Am J Psychiatry 2019;176:67-76.

72 Wechsler D. Manual for the Wechsler abbreviated intelligence scale (WASI). San Antonio, TX: The Psychological Corporation, 1999.

73 Kaufman J, Birmaher B, Brent D. Schedule for affective disorders and schizophrenia for school-age Children-Present and lifetime version (K-SADS-PL): initial reliability and validity data. J Am Acad Child Adolesc Psychiatry 1997;36:980-8.

74 Wiggins JL, Brotman MA, Adleman NE. Neural correlates of irritability in disruptive mood dysregulation and bipolar disorders. Am J Psychiatry 2016;173:722-30.

75 Goodman R, Ford T, Richards $\mathrm{H}$, et al. The development and wellbeing assessment: description and initial validation of an integrated assessment of child and adolescent psychopathology. J Child Psychol Psychiatry 2000;41:645-55.

76 Constantino J, Gruber C. Social responsive scale (SRS) manual. Los Angeles, CA: Western Psychological Services, 2005.

77 Rutter M, Bailey A, Lord C. SCQ: social communication questionnaire. Los Angeles: Western Psychological Services, 2003.

78 Bishop D. Children's communication checklist. 2nd edn. London: The Psychological Corporation, 2003

79 Onken LS, Carroll KM, Shoham V, et al. Reenvisioning clinical science: unifying the discipline to improve the public health. Clin Psychol Sci 2014;2:22-34.

80 Brotman MA, Kircanski K, Gold AL. Exposure-based cognitive behavioral therapy for irritability and disruptive mood dysregulation disorder (DMDD) unpublished manual.

81 Barkley RA. Defiant children: A clinician's manual for assessment and parent training. Guilford Press, 2013.

82 Kazdin AE, Siegel TC, Bass D. Cognitive problem-solving skills training and parent management training in the treatment of antisocial behavior in children. J Consult Clin Psychol 1992:60:733-47.
83 Wampold BE, Minami T, Tierney SC, et al. The placebo is powerful: estimating placebo effects in medicine and psychotherapy from randomized clinical trials. J Clin Psychol 2005;61:835-54.

84 Haller SP, Kircanski K, Stringaris A, et al. The clinician affective reactivity index: validity and reliability of a Clinician-Rated assessment of irritability. Behavior Therapy 2020;51:283-93.

85 Berk M, Ng F, Dodd S, et al. The validity of the cGl severity and improvement scales as measures of clinical effectiveness suitable for routine clinical use. J Eval Clin Pract 2008;14:979-83.

86 Stringaris A, Goodman R, Ferdinando S, et al. The affective reactivity index: a Concise irritability scale for clinical and research settings. J Child Psychol Psychiatry 2012;53:1109-17.

87 Poznanski EO, Mokros HB. Children's depression rating scale, revised (CDRS-R). Los Angeles: Western Psychological Services, 1996.

88 Group RUoPPAS. The pediatric anxiety rating scale (pars): development and psychometric properties. J Am Acad Child Adolesc Psychiatry 2002;41:1061-9.

89 Shaffer D, Gould MS, Brasic J. A children's global assessment scale (CGAS). Arch Gen Psychiatry 1983;40:1228-31.

90 Birmaher B, Brent DA, Chiappetta L, et al. Psychometric properties of the screen for child anxiety related emotional disorders (scared): a replication study. J Am Acad Child Adolesc Psychiatry 1999;38:1230-6.

91 Kay SR, Wolkenfeld F, Murrill LM. Profiles of aggression among psychiatric patients. I. nature and prevalence. J Nerv Ment Dis 1988; 176:539-46.

92 Angold A, Costello E. Mood and feelings questionnaire (MFQ). Durham, NC: Developmental Epidemiology Program, Duke University, 1987.

93 Kircanski K, Thompson RJ, Sorenson J, et al. Rumination and worry in daily life: examining the naturalistic validity of theoretical constructs. Clin Psychol Sci 2015;3:926-39.

94 Smith AR, Kircanski K, Brotman MA, et al. Advancing clinical neuroscience through enhanced tools: pediatric social anxiety as an example. Depress Anxiety 2019;36:701-11.

95 Webb CA, DeRubeis RJ, Amsterdam JD, et al. Two aspects of the therapeutic alliance: differential relations with depressive symptom change. J Consult Clin Psychol 2011;79:279-83.

96 Strunk DR, Brotman MA, DeRubeis RJ. The process of change in cognitive therapy for depression: predictors of early inter-session symptom gains. Behav Res Ther 2010;48:599-606.

97 Stoddard J, Hsu D, Reynolds RC, et al. Aberrant amygdala intrinsic functional connectivity distinguishes youths with bipolar disorder from those with severe mood dysregulation. Psychiatry Res $2015 ; 231: 120-5$

98 White LK, Britton JC, Sequeira S, et al. Behavioral and neural stability of attention bias to threat in healthy adolescents. Neuroimage 2016;136:84-93.

99 Hermoye L, Saint-Martin C, Cosnard G, et al. Pediatric diffusion tensor imaging: normal database and observation of the white matter maturation in early childhood. Neuroimage 2006;29:493-504.

100 Soares JM, Marques P, Alves V, et al. A hitchhiker's guide to diffusion tensor imaging. Front Neurosci 2013;7:31.

101 Rich BA, Carver FW, Holroyd T, et al. Different neural pathways to negative affect in youth with pediatric bipolar disorder and severe mood dysregulation. J Psychiatr Res 2011;45:1283-94.

102 Rich BA, Holroyd T, Carver FW, et al. A preliminary study of the neural mechanisms of frustration in pediatric bipolar disorder using magnetoencephalography. Depress Anxiety 2010;27:276-86.

103 Rich BA, Schmajuk M, Perez-Edgar KE, et al. Different psychophysiological and behavioral responses elicited by frustration in pediatric bipolar disorder and severe mood dysregulation. AJP 2007;164:309-17.

104 Deveney CM, Connolly ME, Haring CT, et al. Neural mechanisms of frustration in chronically irritable children. Am J Psychiatry 2013:170:1186-94.

105 Hommer RE, Meyer A, Stoddard J, et al. Attention bias to threat faces in severe mood dysregulation. Depress Anxiety 2014;31:559-65.

106 MacLeod C, Mathews A, Tata P. Attentional bias in emotional disorders. J Abnorm Psychol 1986;95:15-20.

107 Van Bockstaele B, Lamens L, Salemink E, et al. Reliability and validity of measures of attentional bias towards threat in unselected student samples: seek, but will you find? Cogn Emot 2020;34:217-28.

108 White SF, Tyler PM, Erway AK, et al. Dysfunctional representation of expected value is associated with reinforcement-based decisionmaking deficits in adolescents with conduct problems. J Child Psychol Psychiatr 2016;57:938-46. 
109 Jarrett MA, Ollendick TH. Treatment of comorbid attention-deficit/ hyperactivity disorder and anxiety in children: a multiple baseline design analysis. J Consult Clin Psychol 2012;80:239-44.

110 Lumpkin PW, Silverman WK, Weems CF, et al. Treating a heterogeneous set of anxiety disorders in youths with group cognitive behavioral therapy: a partially nonconcurrent multiplebaseline evaluation. Behav Ther 2002;33:163-77.

111 Faul F, Erdfelder E, Lang A-G, et al. G*Power 3: a flexible statistical power analysis program for the social, behavioral, and biomedical sciences. Behav Res Methods 2007;39:175-91.
112 Gupta S. Intention-To-Treat concept: a review. Perspect Clin Res 2011;2:109.

113 Diggle P, Liang K-Y, Zeger SL. Analysis of longitudinal data. Oxford New York: Clarendon Press; Oxford University Press, 1994.

114 Jacobson NS, Truax P. Clinical significance: a statistical approach to defining meaningful change in psychotherapy research. J Consult Clin Psychol 1991;59:12-19.

115 Tottenham N, Tanaka JW, Leon AC, et al. The NimStim set of facial expressions: judgments from untrained research participants. Psychiatry Res 2009;168:242-9. 